\title{
Latent Tuberculosis Infection
}

\author{
An Expert Interview with Jean-Pierre Zellweger
}

TB Competence Center, Swiss Lung Association, Berne, switzerland

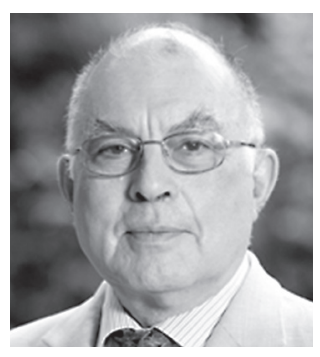

DOl: https://doi.org/10.17925/ERPD.2018.4.1.21

\section{Jean-Pierre Zellweger}

Jean-Pierre Zellweger is a Swiss pulmonary physician, with clinical experience in the management of chronic respiratory diseases, smoking cessation and tuberculosis. He was senior consultant at the University Hospital Medical Policlinic (TB department) in Lausanne. He is now medical adviser for the Swiss Lung Association, interacting with Swiss and international organisations involved in the management of tuberculosis.

\section{Keywords}

Tuberculosis, LTBI, screening, infection

Disclosure: Jean-Pierre Zellweger has

received speaker's fees from Qiagen. He is a member of the journal's editorial board.

Review Process: This is an expert interview and, as such, has not undergone the journal's standard peer review process.

Authorship: The named author meets the International Committee of Medical Journal Editors (ICMJE) criteria for authorship of this manuscript, takes responsibility for the integrity of the work as a whole, and has given final approval for the version to be published.

Open Access: This article is published under the Creative Commons Attribution Non-commercial License, which permits any non-commercial use, distribution, adaptation, and reproduction provided the original author and source are given appropriate credit. (c) The Author 2018.

Received: 11 July 2018

Published Online: 5 September 2018

Citation: European Respiratory \& Pulmonary Diseases. 2018:4(1):21-2

Corresponding Author: Jean-Pierre Zellweger, TB Competence Center, Swiss Lung Association, Chutzenstrasse 10, 3007 Berne, Switzerland.

E: zellwegerip@swissonline.ch

Support: No funding was received in

the publication of this article.
T uberculosis (TB) is a major global public health problem and is the leading cause of death linked to a single pathogen, ranking above human immunodeficiency virus (HIV). ${ }^{1}$ Clinically, TB has been categorised as active disease (patients who are generally symptomatic and may be infectious if pulmonary involvement is present) and latent infection (asymptomatic and not infectious, but at variable risk for progression to active TB disease). It is increasingly being recognised that latent TB infection (LTBI) reflects diverse responses to infection with Mycobacterium tuberculosis and may lead to heterogeneous clinical outcomes. In an expert interview, Jean-Pierre Zellweger discusses the latest World Health Organisation (WHO) guidelines on the management of LTBI.

\section{Q. How prevalent is latent tuberculosis infection around the world?}

LTBI is the stage at which a person has been infected by M. tuberculosis but has not developed the disease. These people develop an immune reaction which can be detected by an indirect test (tuberculin skin test or interferon-gamma release assay), but have no symptoms and cannot transmit the disease. ${ }^{2}$ A minority of those infected will develop TB in the future, mostly during the first 2 years after infection, but the majority will not; this is possibly because they are able to control the infection or they have successfully eliminated the mycobacteria from the organism. The risk of developing TB depends on the quality of the immune defence mechanisms, the persistence of living mycobacteria in the organism, the delay since infection and age. Young children, immunocompromised people and those in recent contact of a case of pulmonary TB have the highest risk of reactivation.

The global prevalence of LTBI broadly reflects the local prevalence of TB and is estimated to be between one-quarter and one-third of the world population, increasing with age. ${ }^{3}$ Those with documented contact with a case of transmissible TB are at the highest risk of infection (up to $50 \%$ chance). In low-incidence countries, the prevalence of infection in the general population is approximately $1 \%$ among young adults, ${ }^{4}$ but can increase to $8 \%$ in elderly adults, ${ }^{5}$ and up to $25 \%$ in adult migrants from high-incidence countries. ${ }^{6}$

In infected persons, the risk of TB development can be decreased by a preventive treatment.

\section{Q. Could you give us a brief summary of the latest WHO guidelines on the management of latent tuberculosis infection?}

All cases of TB arise from people who have been infected and could not control the infection. Therefore, controlling the infection with a preventive treatment could decrease the pool of future TB cases and contribute to the progress towards elimination of TB. ${ }^{7}$ As global detection and 
treatment of all those infected is not feasible (but has been attempted in the past), screening of groups at risk and offering preventive treatment to infected individuals is now considered part of the WHO'S End TB Strategy; in addition to the detection and treatment of active cases, which remains the first priority. ${ }^{8}$

In 2015, WHO issued new guidelines defining the groups in which screening and preventive treatment is considered to be cost-effective; these are contacts of cases of transmissible forms of pulmonary TB (all ages), and immunocompromised people. If the local resources are sufficient, further groups to be considered for screening and preventive treatment are prisoners, migrants from high-burden countries, homeless people, illicit drug users and exposed healthcare workers.9 Persons with incipient TB at the time of screening should receive a full course of anti-tuberculous treatment. Recently, an update of the guidelines has been issued, in which the WHO insists that all national TB guidelines should consider the integration of screening for $L T B I$ and preventive treatment for those with the highest risk of developing TB within the framework of routine activities. ${ }^{10}$ The screening procedures, the selection of the preventive treatment and the management of contacts of multidrug-resistant TB cases is also addressed in this document.

\section{Q. What are the most important changes compared with previous guidelines?}

In previous guidelines (for instance the 2012 guidelines on contact investigation ${ }^{11}$, the main aim of contact investigation was the detection of secondary cases of TB among close contacts and the identification of young children and people who are immunocompromised; these groups were the only people considered eligible for a preventive treatment (with or without the documentation of infection). In the current guidelines, all recent contacts (regardless of age) are eligible for screening and, if found infected, should receive a preventive treatment unless contra-indicated. The increase in cost of systematic screening of contacts is compensated by the fact that only contacts with a documented infection are eligible for a preventive treatment. Uninfected contacts are considered at very low risk of TB development, unless immunocompromised or below the age of 5 years.

Screening for LTBI and preventive treatment for other risk groups, such as prisoners, migrants and healthcare workers, can also be considered, depending the local situation and the available resources.

\section{Q. Which groups are most at risk of LTBI and should be targeted for screening?}

The risk of infection depends on the duration of unprotected exposure to a source of TB transmission (usually a patient with smear-positive pulmonary TB) and the concentration of mycobacteria in the air. ${ }^{12}$ Therefore, close contacts of patients with pulmonary TB should be screened and offered a preventive treatment, if found infected.

The risk of developing TB, once infected, is highest in people who are immunocompromised and young children (below the age of 5 years). These groups should also be submitted to screening if they live in or originate from an environment with a risk of exposure to TB. Prisoners, migrants and healthcare workers may be exposed to TB, depending on the local living or working conditions and the local prevalence of TB. The decision to screen should take into account the local risk and the available resources.

\section{Q. How cost-effective are these recommendations?}

Contact investigations and provision of preventive treatment of those who are infected, young children and immunocompromised persons has been demonstrated as cost-effective. . $^{13,14}$ For migrants, screening and provision of preventive treatment is considered as cost-effective at least in young people originating from high-burden regions. ${ }^{15,16}$

As tests for LTBI do not indicate who will develop TB in the future (Iow positive predictive value), the prescription of a preventive treatment should target people with the highest risk of TB and the highest benefit of treatment, taking into consideration individual factors such as intensity of exposure and quality of the immune defence mechanisms. This can improve the selection of eligible candidates for preventive treatment and contribute to the decrease in the number of infected people submitted to an unnecessary treatment. ${ }^{17,18}$

One of the criticisms of the new policy of screening and preventive treatment for those who are infected is the fact that the effectiveness of the policy depends largely on the adherence to the preventive treatment, which may be high in some groups but very low and unsatisfactory in others. ${ }^{19}$ Therefore, efforts should be made to select the preventive treatment which offers the highest chance of being followed to the end. Short-term treatment regimens (for instance 3 months of isoniazid and rifampicin or 3 months of weekly rifapentine and isoniazid) are well tolerated and yield better adherence than traditional treatments with 6 or 9 months of isoniazid.
1. World Health Organisation (WHO).Global tuberculosis report 2017. Available at: www.who.int/tb/publications/global_report/ en/ (accessed 20 August 2018).

Mack U, Migliori GB, Sester M, et al. LTBI: latent tuberculosis infection or lasting immune responses to M. tuberculosis? infection or lasting immune responses to M. tuberculosis?
A TBNET consensus statement. Eur Respir J. 2009;33:956-73. A TBNET Consensus statement. Eur Respir J. 2009:33:956-73.
Houben RM, Dodd PJ. The global burden of latent tuberculosis infection: a re-estimation using mathematical modelling. PLOS Med. 2016;13:e1002152

4. Mancuso JD, Diffenderfer JM, Ghassemieh BJ, et al. The prevalence of latent tuberculosis infection in the United States Am J Respir Crit Care Med. 2016;194:501-9.

5. Hinks TS, Varsani N, Godsiff DT, et al. High background rates of positive tuberculosis-specific interferon-gamma release assays in a low prevalence region of UK: a surveillance study. BMC Infect Dis. 2012;12:339.

6. Pareek M, Watson JP, Ormerod LP, et al. Screening of immigrants in the UK for imported latent tuberculosis: a multicentre in the UK for imported latent tuberculosis: a multicentre
cohort study and cost-effectiveness analysis. Lancet Infect Dis. 2011:11:435-44.

7. Esmail $H$, Barry CE 3rd, Young DB, Wilkinson RJ. The ongoing challenge of latent tuberculosis. Philos Trans R Soc Lond B Biol Sci. 2014;369:20130437.

8. Lonnroth K, Raviglione M. The WHO's new End TB Strategy in the post-2015 era of the Sustainable Development Goals. Trans $R$ Soc Trop Med Hyg. 2016;110:148-50
9. World Health Organization. Guidelines on the management of latent tuberculosis infection. WHO; 2015. Report No. WHO/ of latent tuberculosis infection. WHO; 2015. Report No. WHO/
HTM/TB/2015.01. Available at: www.who.int/tb/publications/ Itbi_document_page/en/ (accessed 16 August 2018).

10. World Health Organization. Latent tuberculosis infection. Updated and consolidated guidelines for programmatic management. Geneva: World Health Organization; 2018. Report No. WHO/CDS/TB/2018.4. Available at: www.who.int/tb/ publications/2018/latent-tuberculosis-infection/en/ (accessed 16 August 2018).

11. World Health Organization. Recommendations for investigating contacts of persons with infectious tuberculosis in low- and middle-income countries. Geneva; 2012. Report No. WHO/HTM TB/2012.9. Available at: www.who.int/tb/publications/2012 contact investigation2012/en/ (accessed 16 August 2018).

12. Riley RL. Transmission and environmental control of tuberculosis. In: Reichman LB, Hershfield ES (eds). Tuberculosis. tuberculosis. In: Reichman LB, Hershfield ES (eds). Tuberculosis.
A Comprehensive International Approach. Vol. 66. New York: A Comprehensive International Ap

13. Diel R, Nienhaus A, Schaberg T. Cost-effectiveness of isoniazid chemoprevention in close contacts. Eur Respir J. 2005;26:465-73.

14. Diel R, Lampenius $N$, Nienhaus A. Cost effectiveness of preventive treatment for tuberculosis in special high-risk populations. Pharmacoeconomics. 2015;33:783-809.

15. Zenner D, Hafezi H, Potter J, et al. Effectiveness and cost- effectiveness of screening migrants for active tuberculosis and latent tuberculous infection. Int I Tuberc Lung Dis. 2017;21:965-76.

16. Zammarchi L, Casadei G, Strohmeyer M, et al. A scoping review of cost-effectiveness of screening and treatment for latent tuberculosis infection in migrants from highfor latent tuberculosis infection in migrants from high-
incidence countries. BMC Health Serv Res. 2015;15:412. incidence countries. BMC Health Serv Res. 2015;15:412.
7. Sester M, van Crevel R, Leth F, Lange C. Numbers needed to treat to prevent tuberculosis. Eur Respir J. 2015;46:1836-8.

18. Saunders MJ, Wingfield T, Tovar MA, et al. A score to predict and stratify risk of tuberculosis in adult contacts of tuberculosis index cases: a prospective derivation and external validation cohort study. Lancet Infect Dis. 2017;17:1190-9.

19. Alsdurf $\mathrm{H}$, Hill PC, Matteelli $A$, et al. The cascade of care in diagnosis and treatment of latent tuberculosis infection: a systematic review and meta-analysis. Lancet Infect Dis. 2016;16:1269-78.

20. Fox GJ, Dobler CC, Marais BJ, Denholm JT. Preventive therapy for latent tuberculosis infection-the promise and the challenges Int I Infect Dis. 2017:56:68-76.

21. Assefa $Y$, Assefa $Y$, Woldeyohannes $S$, et al. 3-month daily rifampicin and isoniazid compared to 6- or 9-month isoniazid for treating latent tuberculosis infection in children and adolescents less than 15 years of age: an updated systematic review. Eur Respir J. 2018;52: 1800395. 